\title{
Ratio of Monocytes to Lymphocytes in Peripheral Blood in Children Diagnosed with Active Tuberculosis
}

\author{
[i] Ayça Demir, 䧃Mine Düzgöl, 回Nuri Bayram, 回Ahu Kara, 四Hurşit Apa, 四 Ilker Devrim \\ University of Health Sciences, Dr. Behçet Uz Child Diseases and Surgery Training and Research Hospital, Clinic of Child Health and Diseases, İzmir, \\ Turkey
}

\begin{abstract}
Aim: The ratio of monocytes to lymphocytes (ML) could reflect an immunity to Mycobacterium tuberculosis (TB). The objective of this study was to evaluate the relationship between the ratio of $\mathrm{ML}$ and the clinical status of patients with active TB.

Materials and Methods: This was a retrospective review of data collected from the clinical database of the Behcet Uz Children's Research Hospital. One hundred thirty-eight patients were diagnosed with pulmonary and extra-pulmonary TB from January 2006 to January 2015 . White blood cell count, absolute monocyte and absolute lymphocyte counts, the ML ratio, erythrocyte sedimentation rate (ESR) and C-reactive protein (CRP) were compared between extra-pulmonary and pulmonary TB cases. Pre-treatment and after treatment values of the parameters were also compared in both of the groups.

Results: A total of 138 patients were diagnosed as having pulmonary or extra-pulmonary TB during the study period. No significant difference between pulmonary and extra-pulmonary TB was present regarding white blood cell count, absolute $M L$, ESR and CRP ( $p>0.05)$. In patients with pulmonary TB and extra-pulmonary TB, a significant decrease in white blood cell count, absolute monocyte count, ESR and CRP values after treatment compared to pretreatment was observed $(p<0.05)$. The $M L$ ratio was not significantly different in the extra-pulmonary TB group $(p>0.05)$ while a significant difference was present between the pre- and post-treatment groups in pulmonary TB $(p=0.000)$.

Conclusion: The hematological markers including the ML ratio were found to be more useful for monitoring the response of TB therapy, rather than as a differential diagnosis of pulmonary TB from extra-pulmonary TB.
\end{abstract}

Keywords: Extra-pulmonary tuberculosis, pulmonary tuberculosis, ratio of monocyte to lymphocyte counts, children

\section{Introduction}

Tuberculosis (TB) remains a major global health problem affecting millions of people annually. It is the second leading cause of death from infectious disease following human immunodeficiency virus (HIV) worldwide (1). TB is still a major health problem for children as well as adults. Although children constitute $5 \%$ of the TB population in low-burden countries, it is reported to be as high as 20-40\% in other countries (2-5). Globally, there were an estimated
8.6 million new cases of TB in 2013 and 1.3 million deaths (6).

Knowledge about the hematological manifestations of Mycobacterium TB infection is important to provide insight into its pathogenesis. Myeloid-specific cells have been known to serve as host cells for Mycobacterium TB growth and lymphoid cells are thought to be the major effector cells in TB immunity. Given the central role of monocytes and lymphocytes in the induction of immune responses, their levels (hereafter termed "ML ratio") in peripheral blood might be expected to reflect the state

\section{Address for Correspondence}

Nuri Bayram, Dr. Behçet Uz Children University of Health Sciences, Dr. Behçet Uz Child Diseases and Surgery Training and Research Hospital, Clinic of Child Health and Diseases, izmir, Turkey Phone: +90 2324116194 E-mail: nuribayram@gmail.com ORCID: orcid.org/0000-0003-1802-2518

Received: 18.10 .2018 Accepted: 10.06 .2019

${ }^{\circ}$ Copyright 2020 by Ege University Faculty of Medicine, Department of Pediatrics and Ege Children's Foundation

The Journal of Pediatric Research, published by Galenos Publishing House. 
of an individual's immunity to the infection. In a recent clinical analysis of peripheral blood mononuclear cells from a cohort of South African infants, the relative ratio of monocytes to lymphocytes at the start of monitoring was shown to predict the risk of developing TB disease during follow-up (7).

The ratio of monocytes to lymphocytes $(M L)$ in peripheral blood correlated with the extent of TB in both rabbits and humans (8). The number of studies were small and the strength of the conclusions that could be reached in humans seemed to be modest. There was no strong evidence that the $M L$ ratio was affected by Mycobacterium TB infection in humans.

In this study, we reviewed our experience of children with active TB admitted into a tertiary hospital over a 9-year period in Turkey.

We aimed to evaluate the relationship between the ratio of monocytes to lymphocytes, the clinical status of patients with active TB and how the ML ratio could be affected by TB or ongoing anti-TB treatment. This study hypothesized that ML could be a marker for TB in countries with limited resources.

\section{Materials and Methods}

\section{Study population and ethics statement}

Data from all subjects were collected retrospectively from the clinical database of the Pediatric Infectious Disease Department in Behcet Uz Children's Research Hospital, between January 2006 and January 2015. This study was approved by the Ethics Committee of Behcet Uz Children's Research Hospital, and was in compliance with national legislation and the Declaration of Helsinki guidelines. Written patient consent was obtained according to institutional guidelines.

\section{Clinical data base}

We obtained information about patients from archive records retrospectively. The demographic characteristics of the patients [age, gender, complaints, physical examination, contact history with Mycobacterium tuberculosis, treatment), laboratory tests (white blood cell (WBC) count, absolute monocyte counts (AMC) and absolute lymphocyte counts (ALC), monocyte / lymphocyte (ML) ratio, erythrocyte sedimentation rate (ESR), C-reactive protein (CRP), gastric fluid / sputum / bronchoalveolar lavage / biopsy cultures, polymerase chain reaction (PCR) and imaging methods such as tomography and chest radiography] were noted from patient files and electronic database. Patients who had comorbid disease and immunodeficiency were excluded from the study.

\section{Statistical Analyses}

A retrospective cohort study was planned as statistical data using SPSS 20 (Statistical Package for Social Sciences; v20; SPSS Inc, Chicago, USA). Parametric methods were used to analyze the data with normal distribution, nonparametric methods were used to analyze the data and categorical data which did not conform to the normal distribution. Evaluation of normalization was determined according to Kolmogorov-Smirnov analysis. Numerical data was calculated as mean \pm standard deviation or median, nominal number ( $\mathrm{n}$ ) and percentage (\%). Student t-test and Mann-Whitney $U$ test (in nonparametric conditions) were used in comparisons of two independent groups. Nominal data rate portions were compared with chi-square test. For the comparison of data before and after treatment, the dependent student group t-test was used. In nonparametric conditions Wilcoxon signed-rank test was used. In this study, $\mathrm{p}<0.05$ was considered statistically significant.

\section{Results}

\section{Characteristics of the study population}

A total of 138 patients were diagnosed as pulmonary or extra-pulmonary TB during the period of January 2006 to January 2015.

Demographic and clinical characteristics of the study sample

There were 138 individuals enrolled in the study: 103 (74.6\%) patients were diagnosed as pulmonary TB, 17 patients as extra-pulmonary TB. Eighteen patients who had both extra-pulmonary and pulmonary TB were excluded. The distribution of cases with extra-pulmonary TB was as follows;

In the study, 49 patients were male (40.8\%) and 71 patients were female (59.2\%). The median age was 10 years old ranging from 1.5 months to 18 years.

\section{Laboratory features}

Comparison of laboratory markers in extrapulmonary and pulmonary TB

No significant difference between pulmonary and extrapulmonary TB was present regarding WBC, ALC, AMC, M/L ratio, CRP and ESR in the pre-treatment group ( $p>0.05$; Table I).

Comparison of laboratory markers in the pretreatment and after treatment groups. 
All patients with pulmonary TB had a significant decrease in WBC count $(p=0.000), \operatorname{ALC}(p=0.03), A M C$ $(p=0.000), \operatorname{ESR}(p=0.000)$ and CRP $(p=0.000)$ values after treatment compared with pre-treatment (Table II). In the extra-pulmonary TB group, a significant decrease was achieved regarding ESR, WBC count, $A M C$ and CRP after treatment, however no significant difference was present in ALC in extra-pulmonary TB cases ( $p>0.05$ ) (Table II).
All patients with TB were evaluated before and after treatment and the ML ratio was significantly lower after treatment $(p=0.000)$. The $M L$ ratio was not significantly different in the extra-pulmonary TB group ( $p>0.05)$ while a significant difference was present between the pre- and post-treatment groups in pulmonary TB $(p=0.000)$ (Table II).

Table I. Comparison of laboratory markers in the extrapulmonary and pulmonary tuberculosis groups

\begin{tabular}{|c|c|c|c|}
\hline & $\begin{array}{l}\text { Pulmonary tuberculosis } \\
\text { (median) (min-max) }\end{array}$ & $\begin{array}{l}\text { Extrapulmonary tuberculosis } \\
\text { (median) (min-max) }\end{array}$ & $\mathbf{p}$ \\
\hline White blood cell count $\left(/ \mathrm{mm}^{3}\right)$ & $8,500(3,400-24,350)$ & $8,720(4,010-28,650)$ & $>0.05$ \\
\hline Absolute lymphocyte count (/mm $\left.{ }^{3}\right)$ & $2800(900-12,060)$ & $3,200(1,367-10,124)$ & $>0.05$ \\
\hline Absolute monocyte count $\left(/ \mathrm{mm}^{3}\right)$ & $700(151-3,560)$ & $600(300-3,140)$ & $>0.05$ \\
\hline ESR (mm/hour) & $27(1-122)$ & $31(2-92)$ & $>0.05$ \\
\hline CRP (mg/dL) & $1.1(0.11-13)$ & $0.34(0.1-19)$ & $>0.05$ \\
\hline$M / L$ ratio & $0.22(0-1.7)$ & $0.21(0.08-0.49)$ & $>0.05$ \\
\hline
\end{tabular}

Table II. Comparison of laboratory markers in pretreatment and treatment groups in the extrapulmonary and pulmonary tuberculosis groups

\begin{tabular}{|c|c|c|c|c|}
\hline & $\begin{array}{l}\text { Pulmonary } \\
\text { Tuberculosis }\end{array}$ & $\begin{array}{l}\mathrm{p} \\
\text { value }\end{array}$ & $\begin{array}{l}\text { Extra-pulmonary } \\
\text { Tuberculosis }\end{array}$ & $\begin{array}{l}\mathrm{P} \\
\text { value }\end{array}$ \\
\hline & Median & & Median & \\
\hline \multicolumn{5}{|l|}{ WBC $\left(/ \mathrm{mm}^{3}\right)$} \\
\hline Pretreatment & 8,500 & \multirow[t]{2}{*}{0.000} & 8,720 & 0.044 \\
\hline After treatment & 7,460 & & 6,300 & \\
\hline \multicolumn{5}{|c|}{ Absolute Lymphocyte count $\left(/ \mathrm{mm}^{3}\right)$} \\
\hline Pretreatment & 2,800 & \multirow[t]{2}{*}{0.003} & 3,200 & 0.642 \\
\hline After treatment & 2,610 & & 2,400 & \\
\hline \multicolumn{5}{|c|}{ Absolute Monocyte count $\left(/ \mathrm{mm}^{3}\right)$} \\
\hline Pretreatment & 700 & \multirow[t]{2}{*}{0.000} & 600 & 0.010 \\
\hline After treatment & 530 & & 500 & \\
\hline \multicolumn{5}{|l|}{ ESR (mm/hour) } \\
\hline Pretreatment & 27 & \multirow[t]{2}{*}{0.000} & 31 & 0.010 \\
\hline After treatment & 12 & & 9 & \\
\hline \multicolumn{5}{|l|}{ CRP $(\mathrm{mg} / \mathrm{dL})$} \\
\hline Pretreatment & 1.1 & \multirow[t]{2}{*}{0.000} & 0.34 & \multirow[t]{2}{*}{0.016} \\
\hline After treatment & 0.34 & & 0.34 & \\
\hline \multicolumn{5}{|c|}{ Monocyte/lymphocyte Ratio } \\
\hline Pretreatment & 0.22 & 0.000 & 0.21 & 0.099 \\
\hline After treatment & 0.19 & & 0.20 & \\
\hline
\end{tabular}




\section{Discussion}

The ML ratio in peripheral circulation may reflect an individual's capacity to mount an effective immune response. The $M L$ ratio has been shown to correlate with inhibition of mycobacterial growth in vitro $(9,10)$ and risk is higher among individuals with either a low or high $\mathrm{ML}$ ratio (11). This ratio could herald a previously unknown pathophysiologic change of TB. In the literature, ML ratios were reported to be disrupted with $T B ; M L$ ratios in the extreme percentiles are associated with active TB (12).

New diagnostic strategies for sputum smear-negative tuberculosis are urgently needed. CRP is a non-specific inflammatory protein that is usually elevated in patients with $T B$, but its role in the diagnosis of $T B$ is uncertain (13). In this study, CRP and ESR were found to be higher in patients with active TB and the decrease observed in these parameters after treatment suggested that they could be used for monitoring the treatment of TB.

The ML ratio in peripheral circulation may reflect an individual's capacity to mount an effective immune response. The $M L$ ratio has been shown to correlate with inhibition of mycobacterial growth in vitro $(10,14)$ and risk is higher among individuals with either a low or high ML ratio (11). These results add to evidence supporting that extremes of immunity are associated with TB. This ratio could herald a previously unknown pathophysiologic change of TB. As demonstrated by one study, patients with active TB had a higher or lower ML ratio compared to healthy donors. Healthy donors were mostly in the group with an ML ratio between the $9^{\text {th }}$ and $25^{\text {th }}$ percentile, while patients were mainly in the group with an $\mathrm{ML}$ ratio greater than the $25^{\text {th }}$ percentile or in a group with an ML ratio less than the $9^{\text {th }}$ percentile. In order to further evaluate whether the $\mathrm{ML}$ ratio could be affected by anti-TB therapy, the difference between before treatment and after treatment was analyzed. The results indicated that the high $M L$ ratio decreased, and the low $M L$ ratio increased to be close to the ML ratio of healthy donors. It was suggested that the ML ratio of patients may change with anti-TB therapy and the alteration of the $M L$ ratio may also reflect the effectiveness and phase of therapy (10). In our study, a high ML ratio in peripheral blood before treatment showed activity of $M$. tuberculosis infection and this high $M L$ ratio decreased but not close to the ML ratio of healthy donors in accordance with previous findings $(10,11)$. According to our findings, the ML ratio might be used as a useful marker in children to compare pretreatment and treatment. Wang et al. (12) detected that the median $\mathrm{ML}$ ratio before treatment was found to be 0.21 , in our study, this ratio was 0.36 . This difference was explained by the fact that the lymphocyte count in the pediatric age group is higher than the adult age group. In the previous article focusing on adults, those patients older than 60 years were reported to be more likely to be in the group of ML ratio $<9 \%$ or $\mathrm{ML}$ ratio $>25 \%$ compared to younger adults, suggesting age might be an important factor for the ML ratio (12).

\section{Study Limitations}

Our study has some limitations. This study was a retrospective study and needs to be confirmed by prospective studies with wider participation. All patients were immunocompetent, thus the response of the $M L$ ratio in a specific group such as immunosuppressed patients could not be determined with this study. Another limitation of this study was the fact that there was no control group of healthy patients to compare their values before and after treatment. Therefore, the ML ratio was evaluated as before and after treatment.

\section{Conclusion}

In conclusion, hematological markers including M/L rate were found to be useful for monitoring the response of tuberculosis therapy, rather than as a differential diagnosis of pulmonary tuberculosis from extra-pulmonary tuberculosis.

\section{Ethics}

Ethics Committee Approval: This study was approved by the Ethics Committee of Behcet Uz Children's Research Hospital, and was in compliance with national legislation and the Declaration of Helsinki guidelines.

Informed Consent: Written patient consent was obtained according to institutional guidelines.

Peer-review: Internally peer-reviewed.

\section{Authorship Contributions}

Concept: N.B., H.A., I.D., Design: N.B., Data Collection or Processing: A.D., M.D., A.K., H.A., Analysis or Interpretation: I.D., Literature Search: M.D., Writing: A.D., M.D., N.B., A.K., H.A., I.D.

Conflict of Interest: The authors have no conflicts of interest relevant to this article to disclose.

Financial Disclosure: The authors have no financial relationships relevant to this article to disclose.

\section{References}

1. WHO. Global tuberculosis report 2012. http://apps.who.int/ iris/bitstream/10665/75938/1/ 9789241564502eng.pdf

2. Nelson L), Wells CD. Global epidemiology of childhood tuberculosis. Int J Tuberc Lung Dis 2004;8:636-47. 
3. Marais BJ, Hesseling AC, Gie RP, Schaaf HS, Beyers N. The burden of childhood tuberculosis and the accuracy of communitybased surveillance data. Int I Tuberc Lung Dis 2006;10:259-63.

4. World Health Organization. A Research Agenda for Childhood Tuberculosis: Improving the Management of Childhood Tuberculosis Within National Tuberculosis Programmes: Research Priorities Based on a Literature Review.Geneva Switzerland: World Health Organization; 2007.

5. Swaminathan S, Rekha B. Pediatric tuberculosis: global overview and challenges. Clin Infect Dis 2010;50(Suppl 3):184-94.

6. WHO;Global tuberculosis report 2013 ,World Health Organization, Geneva, Switzerland (2013).

7. Naranbhai V, Hill AV, Abdool Karim SS, et al. Ratio of monocytes to lymphocytes in peripheral blood identifies adults at risk of incident tuberculosis among HIV-infected adults initiating antiretroviral therapy. I Infect Dis 2014;209:500-9.

8. Rogers PM. A study of the blood monocytes in children with tuberculosis. N Engl / Med 1928;198:740-9.

9. Carpenter E, Fray L, Gormley E. Cellular responses and Mycobacterium bovis BCG growth inhibition by bovine lymphocytes. Immunol Cell Biol 1997;75:554-60.
10. Denis M, Wedlock DN, Buddle BM. Ability of $T$ cell subsets and their soluble mediators to modulate the replication of Mycobacterium bovis in bovine macrophages. Cell Immunol 2004;232:1-8

11. Tobin DM, Roca FJ, Oh SF, et al. Host genotype-specific therapies can optimize the inflammatory response to mycobacterial infections. Cell 2012;148:434-46.

12. Wang J, Yin $Y$, Wang $X$, et al. Ratio of monocytes to lymphocytes in peripheral blood in patients diagnosed with active tuberculosis. Braz / Infect Dis 2015;19:125-31.

13. Wilson D, Badri M, Maartens G. Performance of serum C-reactive protein as ascreening test for smear-negative tuberculosis in an ambulatory high HIV prevalence population. PloS One 2011;6:e15248

14. Carpenter E, Fray L, Gormley E. Cellular responses and Mycobacterium bovis BCG growth inhibition by bovine lymphocytes. Immunol Cell Biol 1997;75:554-60. 